\title{
Ion energy enhancement from TNSA plasmas obtained from advanced targets
}

\author{
L. TORRISI \\ Department of Physics and SdT, Messina University, Messina, Italy \\ (RECEIVED 18 January 2014; ACCEPTED 22 April 2014)
}

\begin{abstract}
Laser generated plasmas from target normal sheath acceleration produce energetic ions from the rear side of the target due to the formation of a high directive electric field. Fast electrons are ejected from the rear side of the target and a successive Coulomb explosion is driven by the fast electrons generating a high electric field of double layer. The ion acceleration is mainly controlled by the laser intensity and by the square of the laser wavelength. Literature reports that at intensities of the order of $10^{18} \mathrm{~W} / \mathrm{cm}^{2}$ and at wavelengths of about $1 \mu \mathrm{m}$ the ion energy is of the order of $5 \mathrm{MeV} /$ nucleon. The use of advanced targets realized with the aim to reduce the surface reflection, to increase the laser absorption coefficient and, with an optimal thickness, to increase the electric field of the double layer, permits to enhance the ion energy acceleration, so that the energy of $5.0 \mathrm{MeV}$ per charge state can be reached at about $10^{16} \mathrm{~W} / \mathrm{cm}^{2}$, as it will be presented and discussed.
\end{abstract}

Keywords: Advanced targets; Laser-generated plasma; Thomson parabola spectrometer; TNSA

\section{INTRODUCTION}

Target normal sheath acceleration (TNSA) method can be employed to generate high ion acceleration from thin films irradiated by pulsed lasers at intensities higher than $10^{15}$ $\mathrm{W} / \mathrm{cm}^{2}$. The energy of the ions driven by the high electric field developed in the rear-side of the thin irradiated targets is proportional to the laser intensity and to the square of the laser wavelength (Hegelich et al., 2006). Values above $10 \mathrm{MeV} /$ nucleon can be obtained at laser intensities above $10^{18} \mathrm{~W} / \mathrm{cm}^{2}$ using infrared wavelengths and thin films (Spencer et al., 2001; Margarone et al., 2012). Not only are the laser intensity and the laser wavelength essential parameters to obtain high ion acceleration, but also the irradiation conditions and the target composition and geometry represent two other important aspects to be optimized in order to enhance the ion acceleration processes.

Laser irradiations are essential because the laser focalization with respect to the target surface, the use of pre-pulse, the influence of the pulse contrast or the use of polarized laser light represents, for example, some key parameters to control linear and non-linear phenomena producing plasma (Torrisi et al., 2008; McKenna et al., 2006). Self-focusing,

Address correspondence and reprint requests to: L. Torrisi, Department of Physics \& SdT, Messina University, V.le S. D'Alcontres 31, 98166 S. Agata, Messina, Italy. E-mail: lorenzo.torrisi@unime.it pre-plasma, and $p$-polarized light, for example, can be employed to enhance the laser energy transferred to the target and consequently to enhance the forward ion acceleration from TNSA experiments.

Target composition and geometry are also essentials to control the composition and the characteristics of the produced plasma. Metals are strong absorbent of the laser light and may produce high electron density, but show high reflectivity and low proton emission; on the contrary, polymers are low absorbent of the laser light, which produce low electron density, but show high proton emission yield. Thus, in order to produce high energetic proton beams, it is necessary to use composed targets containing both metals and polymers, for example, in order to enhance the proton emission in terms of kinetic energy and yield.

"Advanced" targets can be prepared in order to reduce the laser light component not released to the thin target, due to reflections, scattering, and transmitted laser light, increasing the component of the absorbed laser light, which is responsible of the plasma production and of the high electric field driving the ion acceleration. Special morphological surfaces and complex compositions, such as foam, porous and roughness surfaces, and the use of embedded nano-structures in the target producing high absorption effects at specific wavelengths, can be employed to enhance the laser absorption in the thin target. Such surfaces reduce strongly the reflection 
coefficient and the use of nano-structures, such as metallic nano-spheres, increases the absorption coefficient due to resonant absorption effects such as the surface plasmon resonant process (Giulietti \& Gizzi, 1998; Dombi et al., 2009), and increases also the plasma electron density.

The use of these special targets permit to enhance significantly the ion energy acceleration process, as it will be presented and discussed in this article.

\section{EXPERIMENTAL SET-UP}

Experiments were performed at PALS Laboratory in Prague using a Iodine laser operating at $1315 \mathrm{~nm}$ wavelength, $700 \mathrm{~J}$ maximum pulse energy, $300 \mathrm{ps}$ pulse duration, $70 \mu \mathrm{m}$ laser spot on the target surface, with a maximum intensity of about $10^{16} \mathrm{~W} / \mathrm{cm}^{2}$, operating in single pulse. The target holder is movable with an accuracy of \pm 1 micron in the $x$, $y$, and $z$ directions. The scattering chamber operates with a vacuum of $2 \times 10^{-5} \mathrm{mbar}$.

Different investigated advanced targets were based on the use of polymers and metals. Polymers, based on polyethylene and polyvinyl alcohol (PVA), contain nano-structures of $\mathrm{Fe}_{2} \mathrm{O}_{3}, \mathrm{TiH}_{2}$, carbon nano-tubes and metal nano-spheres ( $\mathrm{Ag}$ and $\mathrm{Au}$ spheres with $50-100 \mathrm{~nm}$ in diameter). The nano-structures were embedded using concentrations of the order of $1 \%$ in weight. Targets were prepared using the spin coating deposition method. Metals were used with different atomic numbers, from $\mathrm{Al}$ to $\mathrm{Au}$, and different thickness, from 1 to 40 microns. Some thin metallic films, of the order of $100 \mathrm{~nm}$ in thickness, were deposited in vacuum by electron beam on thin polymeric mylar films $(0.5-10 \mu \mathrm{m}$ in thickness). The targets are planar and their total thickness ranges between 0.5 to $40 \mu \mathrm{m}$.

The metal nano-spheres of $\mathrm{Ag}$ and $\mathrm{Au}$ were obtained by laser ablation in liquid solution using a pulsed $\mathrm{Nd}$ :Yag laser at $1064 \mathrm{~nm}, 3 \mathrm{~ns}$ pulse duration, $10^{9} \mathrm{~W} / \mathrm{cm}^{2}$ intensity operating at $10 \mathrm{~Hz}$ repetition rate. The used concentrations were of $1-5 \mathrm{mg} / 15 \mathrm{ml}$ solution. The surface treatments to modify the surface roughness were obtained with different techniques, such as sandblasting, ion sputtering, acid attack, and pulse laser deposition of thin films.

In order to control the laser focal position (FP) with respect to the target surface, a micrometric optical alignment and an $\mathrm{X}$-ray streak camera were employed. The optical alignment of the target uses a theodolite, magnification lens, and $x, y$, $z$ in vacuum motorized micrometers. The streak camera observes the target from the top using a $50 \mu \mathrm{m}$ pin-hole and records the plasma emitted in backward direction. It is employed with an exposition time of $2 \mathrm{~ns}$ and a resolution time of $30 \mathrm{ps}$; on the axial plane normal to the target its spatial range is 500 micron. Streak camera detects photons approximately from $100 \mathrm{eV}$ up to $10 \mathrm{keV}$.

The energy, the yield, and the angular distribution of the ions emitted in the forward direction from the produced plasma were measured using Thomson parabola spectrometer (TPS), ion collector rings (ICR), SiC detectors and thin absorber films covering track detectors (CR39). ICR and $\mathrm{SiC}$ are employed in time-of-flight (TOF) approach using fast storage oscilloscopes (20 GS/s) (Cutroneo et al., 2012). Such detectors were placed mainly in the forward direction, i.e., behind the thin irradiated target. TPS spectra were acquired with a magnetic field of $0.06 \mathrm{~T}$ and an electrical field of $1.4 \mathrm{kV} / \mathrm{cm}$; the distance between the electric deflection plates and the multi-channel-plates was $16.5 \mathrm{~cm}$. TPS was placed at $0^{\circ}$ along the normal to the target direction and the other detectors at different distances from the target $(0.6-1.6 \mathrm{~m})$ and at different angles $\left(<60^{\circ}\right)$ with respect to the normal to the target direction.

TPS deflects the ions transmitted through a $100 \mu \mathrm{m}$ pinhole at $1 \mathrm{~m}$ distant from the target and uses a multichannel-plate coupled to a phosphorus screen and to a

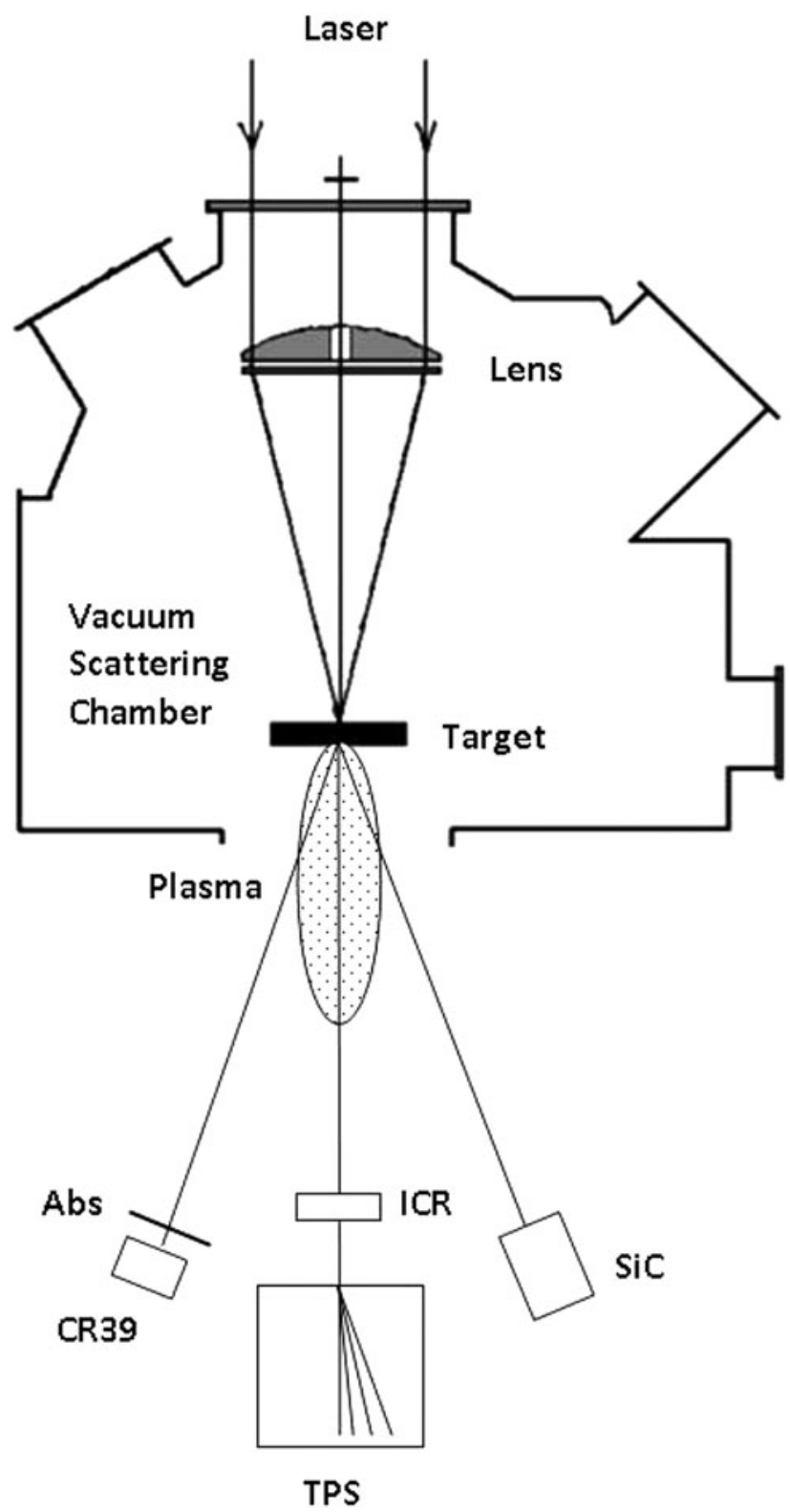

Fig. 1. Scheme of the experimental set-up. 
charge-coupled device camera to record the ion parabolas (Cutroneo et al., 2012). SiC has a surface of $4 \mathrm{~mm}^{2}$ and generally is placed at a distance of $60 \mathrm{~cm}$ from the target.

Figure 1 shows a scheme of the employed experimental set-up. The TPS parabola recognition and the evaluation of the maximum ion energy was obtained using a simulation program based on Opera 3D-Tosca and MathLab code, taking in consideration the charge-to-mass ratios and the values and the real geometry of the used magnetic and electric deflection fields (Opera 3D Tosca Code, 2013).

\section{RESULTS}

Figure 2 shows a typical TPS spectrum of ions emitted irradiating a polyvinylalcohol (PVA) film, 10 micron in thickness, with embedded pure nano-particles of Au 50-100 nm in diameter and $1 \%$ in weight concentration. The spectrum is obtained irradiating the target at $\sim 0^{\circ}$ incidence angle with $500 \mathrm{~J}$ pulse energy with $\mathrm{FP}=-100 \mu \mathrm{m}$. It shows the lower parabola due to the protons deflection and a lot of dense parabolas due to $\mathrm{C}, \mathrm{O}$, and $\mathrm{Au}$ ions at different
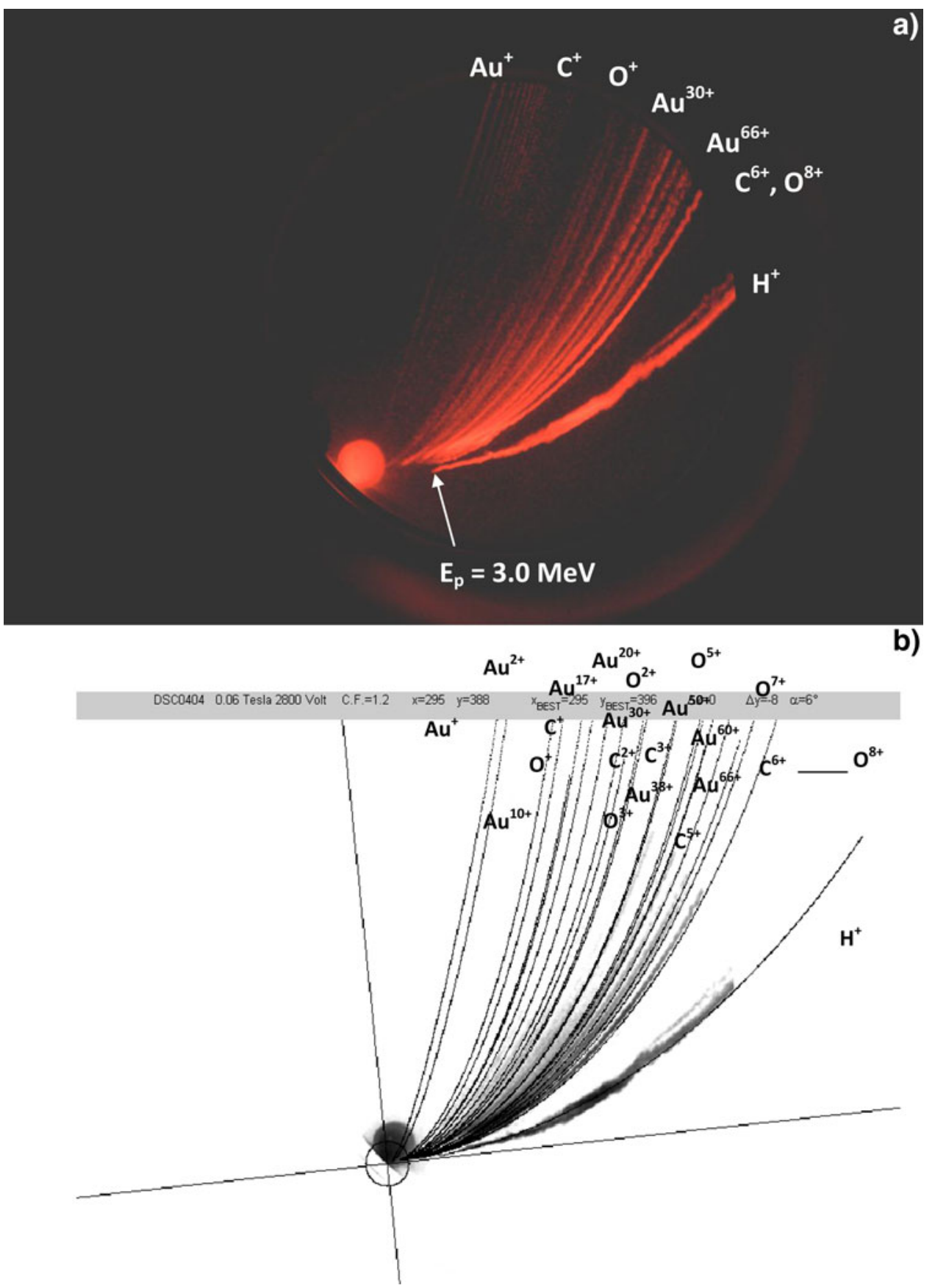

Fig. 2. (Color online) Typical forward $\left(0^{\circ}\right)$ TPS spectrum of Au nano-particles embedded in PVA, $10 \mu \mathrm{m}$ in thickness, irradiated at $500 \mathrm{~J}$ (a) and parabolas recognition study using the Opera 3D-Tosca and MathLab code simulation (b). 
charge states coming from the polymeric resin and from the metallic embedded nano-particles. Some parabolas are coincident due to the same charge-to-mass ratio, such as $\mathrm{C}^{6+}$ and $\mathrm{O}^{8+}$ having the same ratio $1 / 2$. The nearest points of the protons parabola to the zero circle of the undeflected photon radiations are due to the higher energetic protons. Simulations indicate that such proton energy is about 4.0 MeV and that the maximum energy of the other ion species is approximately $4.0 \mathrm{MeV}$ per charge state. The parabola recognition performed with Tosca code permits to identify the parabola due to protons, the six parabolas due to the six charge states of $\mathrm{C}$, the eight charge states of $\mathrm{O}$ and $\mathrm{Au}$ ions, from $\mathrm{Au}^{1+}$ up to a charge state of about $\mathrm{Au}^{66+}$.

This maximum charge state represents a characteristic of the obtained plasma, due to the maximum ionization potential represented by the $\mathrm{Au}^{66+}$ ions corresponding to $7.91 \mathrm{keV}$ (Zschornack et al., 1986). Because no higher charge states of $\mathrm{Au}$ ions are detected, it is possible to assume that the maximum electron energy is about $8 \mathrm{keV}$. Moreover, by assuming the electron energy distribution to be a Boltzmann-like shape, the average electron energy, $\bar{E}$, can be assumed to approach about $1 / 3$ of the maximum energy, i.e., it should be about $2.67 \mathrm{keV}$. Thus, as a first approximation, the possible equivalent electron temperature of the plasma should be about:

$$
\mathrm{kT}_{\mathrm{e}}=\frac{2}{3} \overline{\mathrm{E}}=1.8 \mathrm{keV}
$$

Figure 3a shows a typical $\mathrm{SiC}$ detector-TOF spectrum obtained in the same experimental conditions of TPS spectrum acquisition reported in Figure 2. The spectrum reports, in order of increasing time, the fast photo-peak, the electrons (or Bremsstrahlung) signal, the protons peak, and the large and slower peak due to the convolution of different detected ions emitted from plasma, of which carbons are the faster. The $\mathrm{SiC}$ is placed at $30^{\circ}$ angle with respect to the target normal direction and its distance from the target is $60 \mathrm{~cm}$, thus the initial proton peak at $20 \mathrm{~ns}$ corresponds to protons at a maximum kinetic energy of $4.7 \mathrm{MeV}$. This result, in agreement with the $4.0 \mathrm{MeV}$ protons obtained by TPS measurements, demonstrates that $\mathrm{SiC}$ detector is more sensitive to the high energy of protons with respect to TPS, confirming that semiconductors give a signal proportional to the ion energy (Cutroneo et al., 2012). For comparison, Figure 3b shows a typical spectrum of a similar target without the $\mathrm{Au}$ nano-structures irradiated in the same experimental conditions. In this case, it is possible to observe a protons peak shifted to about $65 \mathrm{~ns}$ which corresponds to a kinetic energy of about $630 \mathrm{keV}$.

Figure 4a shows a typical ICR spectrum relative to the signal acquired in the forward direction at $0^{\circ}$ and at a target distance of $103 \mathrm{~cm}$ placed in front of the TPS. This spectrum is acquired using a laser pulse of $600 \mathrm{~J}$, a FP $=-100 \mu \mathrm{m}$ and 8 micron $\mathrm{Al}$ absorber film placed in front of the ICR detector so that the photo-peak is reduced, heavy ions are absorbed
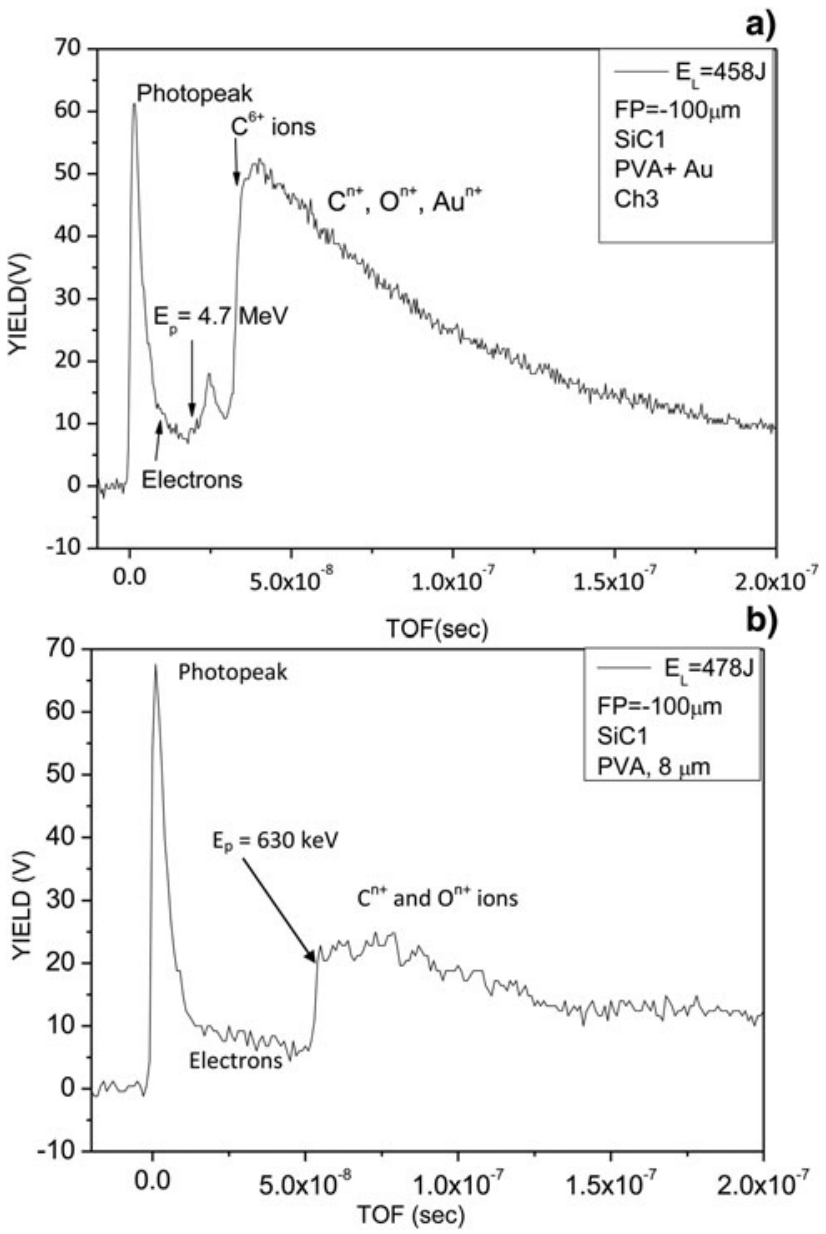

Fig. 3. Typical forward $\left(30^{\circ}\right) \mathrm{SiC}-\mathrm{TOF}$ spectra at $60 \mathrm{~cm}$ distance of $\mathrm{Au}$ nano-particles embedded in PVA, $10 \mu \mathrm{m}$ in thickness, irradiated at $500 \mathrm{~J}$ (a) and of PVA polymer target without nano-particles irradiated in the same experimental conditions (b).

and only energetic protons can be transmitted and detected with a high signal-to-noise ratio. The $\mathrm{Al}$ film absorbs the visible light, protons up to $600 \mathrm{keV}, \mathrm{C}$ ions up to $9 \mathrm{MeV}$, O ions up to $12 \mathrm{MeV}$, and $\mathrm{Au}$ ions up to $35 \mathrm{MeV}$, thus its use in front of the ICR detectors permits a well separation of protons with respect to other ions. The TOF spectrum shows the fast photo-peak due to $\mathrm{X}$-ray detection followed by a peak due to protons detection. The faster protons have a TOF of $33 \mathrm{~ns}$ which corresponds to a kinetic energy of $5.0 \mathrm{MeV}$. This result confirms the measurements of high proton energy performed with TPS and SiC detectors.

CR39 track detectors were placed 150 micron behind the $\mathrm{Al}$ absorber in order to detect only energetic protons, with a kinetic energy higher than $4.3 \mathrm{MeV}$, well separated from heavy ions. Figure $4 \mathrm{~b}$ shows a photo of the optical microscopy of a typical CR39 film detector after the chemical etching for protons track detection. This result confirms that protons with energy higher than $4.3 \mathrm{MeV}$ are emitted from the used advanced thin targets irradiated at PALS.

Reproducible measurements were performed using the same experimental conditions of laser irradiation and 

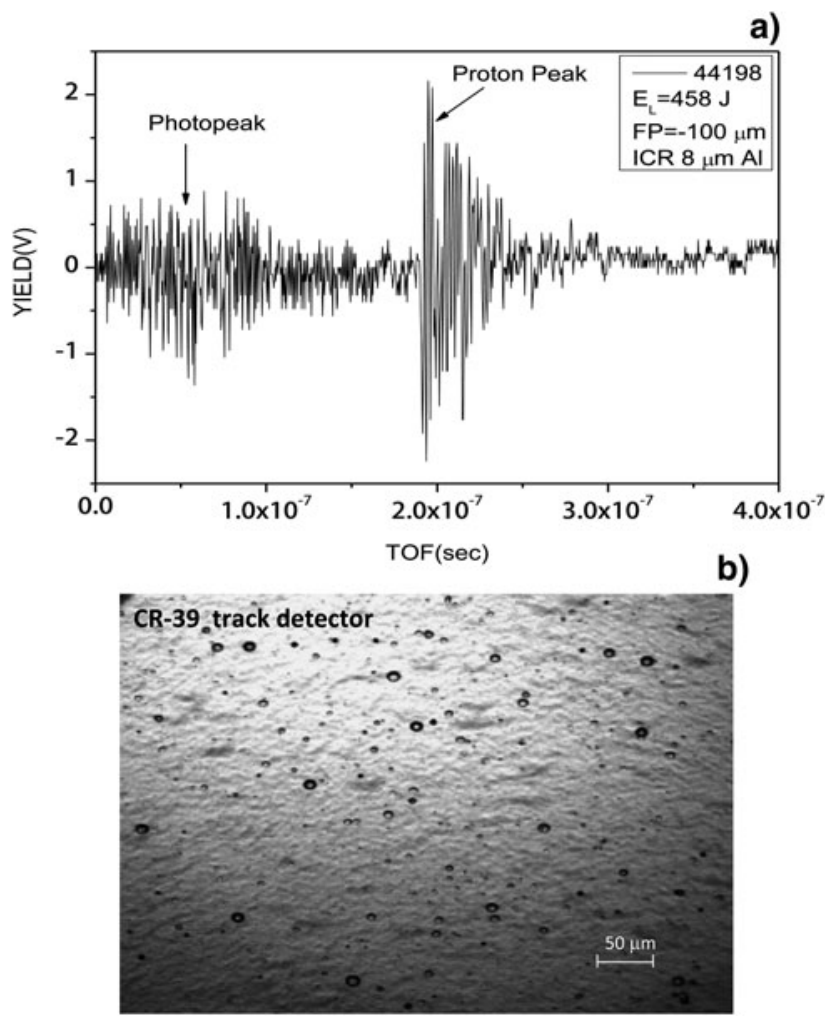

Fig. 4. Typical ICR spectrum relative to the signal acquired in forward direction at $0^{\circ}$ and $103 \mathrm{~cm}$ distance from the target (a) and CR39 track detectors photo placed behind 150 micron $\mathrm{Al}$ absorber after the chemical etching for proton track detection (b).

different prepared advanced targets. Such investigations have indicated that the laser focal position, to maximize the ion acceleration, is crucial, otherwise the maximum ion acceleration decreases. The control of this parameter was obtained using the streak camera $\mathrm{X}$-ray images giving the shape, the dimension, the target surface distance from the plasma core and the temperature gradient of the produced plasma. A typical charge-coupled device image of the X-ray generation in the backward direction for a focal position of $-100 \mu \mathrm{m}$ from the target surface is reported in Figure 5a for the irradiation of pure PVA without nano-particles and in Figure $5 \mathrm{~b}$ for the irradiation of the PVA with embedded Au nano-particles. The comparison of the two images indicates that the backward plasma in the first case (Fig. 5a) is near absent, due to the laser light well transmitted by the thin target. In the second case (Fig. 5b), in presence of metallic Au nano-structures, the laser is well absorbed and the plasma is produced in the backward direction, as observed trough the higher density and clear colors of the plume $\mathrm{X}$-ray image. The two photos have a horizontal scale relative to time duration of $1 \mathrm{~ns}$ and a vertical scale relative to a spatial range of \pm 100 microns. The zero time scale corresponds to the laser shot pulse; the zero distance scale corresponds to the target position. The plasma duration is of about $400 \mathrm{ps}$, i.e., comparable with the laser pulse duration and this

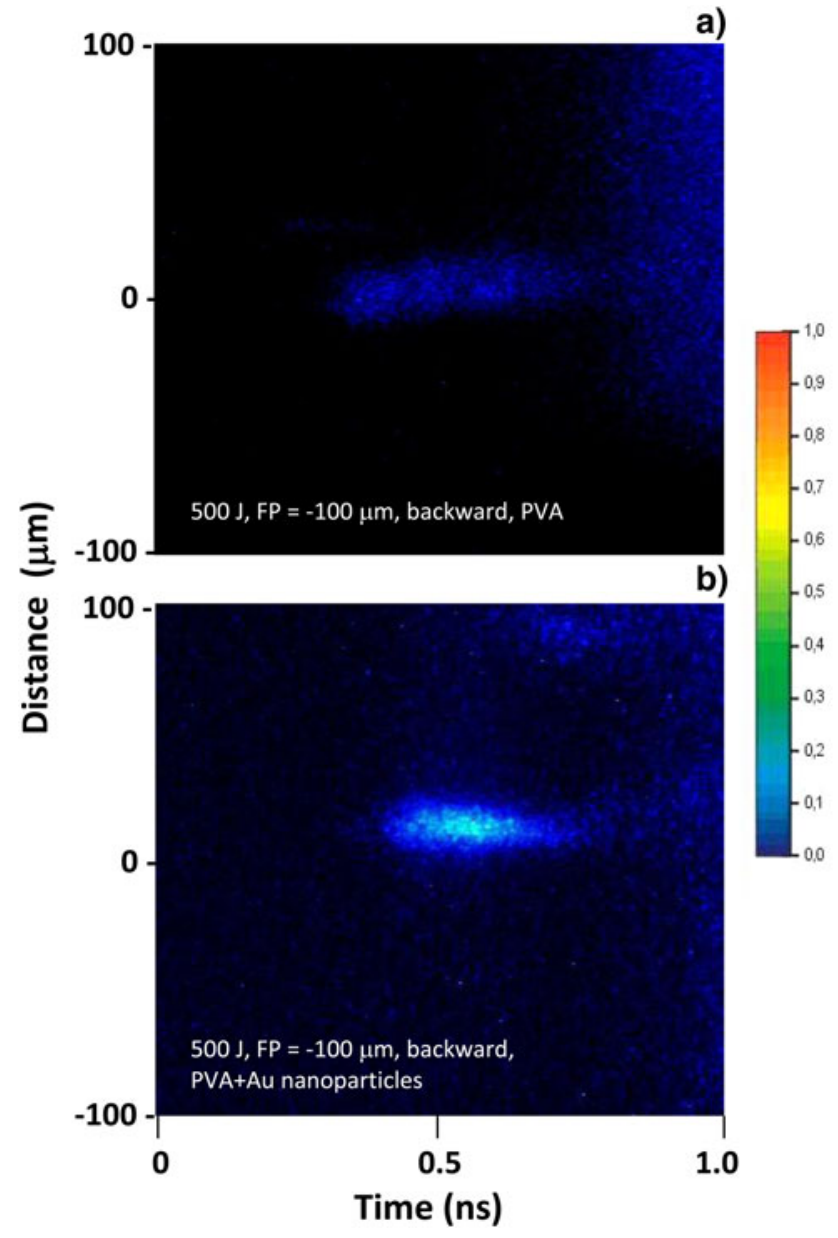

Fig. 5. (Color online) X-ray streak camera image of plasma produced in backward direction during the $500 \mathrm{~J}, \mathrm{FP}=-100 \mu \mathrm{m}$ laser irradiation of pure PVA (a) and of PVA with Au nano-structures (b).

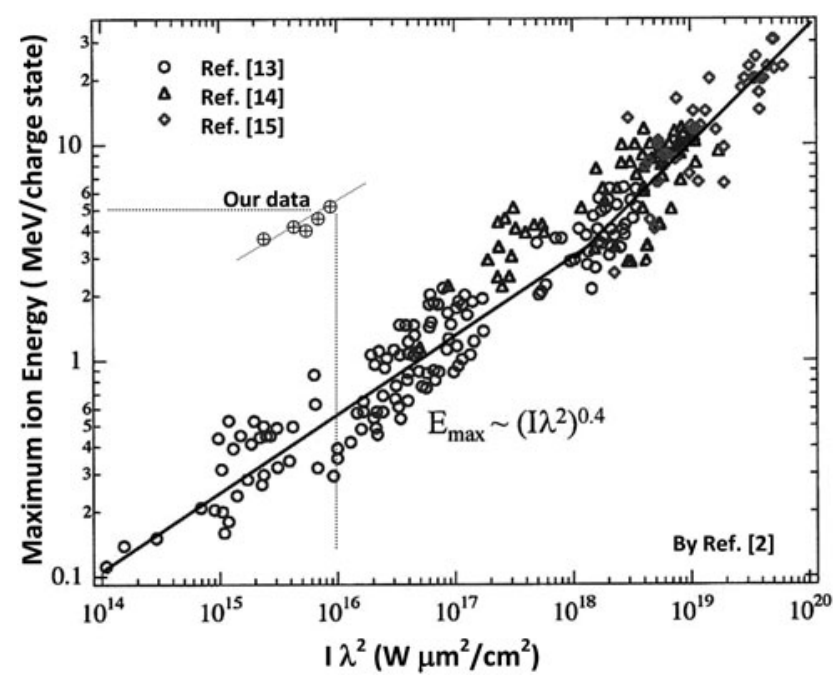

Fig. 6. Comparison between the law scale plot by Spencer et al. (2001) and our measurements obtained using "advanced" targets irradiated at $10^{16} \mathrm{~W} /$ $\mathrm{cm}^{2}$ laser intensity. 
maximum size in the backward direction is about 30 microns. Plasma starts at 100 micron distance from the target surface.

Similar results of proton acceleration at about $5.0 \mathrm{MeV}$ maximum kinetic energy were obtained with Ag nanospheres embedded in PVA with a total target thickness of 10 microns and with $\mathrm{Au}$ nano-spheres embedded in 15 micron PVA thickness, with absorbed hydrogen, was treated with sandblasting to obtain an average roughness of about 10 microns.

\section{DISCUSSION}

The measurements presented in this article demonstrated that, using thin foils composed by PVA resin containing metallic low concentration $\mathrm{Au}$ nano-structures, energetic non-equilibrium plasma is generated in the forward direction. The equivalent electron temperature should be of the order of $1.8 \mathrm{keV}$. For protons, assuming to be in near local thermal equilibrium $\left(k T_{\mathrm{e}} \approx k T_{\mathrm{i}}\right)$, and the thermal velocity is about:

$$
\mathrm{v}_{\mathrm{T}}=\sqrt{\frac{3 \mathrm{kT}}{\mathrm{m}_{\mathrm{p}}}}=7.2 \times 10^{5} \mathrm{~m} / \mathrm{s} .
$$

Such result is in agreement with the X-ray streak camera image that doesn't show significant lateral expansion. Assuming the lateral plasma expansion to be about $3 \mu \mathrm{m}$, the expansion velocity in the $400 \mathrm{ps}$ is $7.5 \times 10^{5} \mathrm{~m} / \mathrm{s}$.

The plasma protons expansion velocity in vacuum can be calculated by the equation:

$$
\mathrm{v}_{\mathrm{K}}=\sqrt{\frac{\gamma \mathrm{kT}}{\mathrm{m}_{\mathrm{p}}}}=5.4 \times 10^{5} \mathrm{~m} / \mathrm{s},
$$

where $\gamma$ is the adiabatic coefficient and $m_{\mathrm{p}}$ is the proton mass. The Coulomb interactions contribute to the ion velocity more than one order of magnitude with respect to the thermal interactions, in fact its value for $5 \mathrm{MeV}$ protons is:

$$
\mathrm{v}_{\mathrm{C}}=\sqrt{\frac{2 \mathrm{E}}{\mathrm{m}_{\mathrm{p}}}}=3.1 \times 10^{7} \mathrm{~m} / \mathrm{s} .
$$

Ions are accelerated up to about $5.0 \mathrm{MeV}$ per charge state along the direction normal to the target surface, demonstrating that the mechanism of TNSA occurs also in a regime of the order of $10^{16} \mathrm{~W} / \mathrm{cm}^{2}$ laser intensity using a pulse duration of about $300 \mathrm{ps}$, in contradiction to many other articles indicating a validity for intensities above $10^{18} \mathrm{~W} / \mathrm{cm}^{2}$ (Spencer et al., 2001; Badziak et al., 2005). This mechanism was verified also by TPS measurements of energetic electrons, obtained using an inverse electric field and a weak magnetic deflection field of only $50 \mathrm{G}$. Fast electrons with a parabola point from $2 \mathrm{MeV}$ up to $8 \mathrm{MeV}$ kinetic energy were measured.

The maximum ion acceleration was obtained only with target thickness of about 10-15 microns, flat surfaces, laser pulse energy of 500-600 J, $1315 \mathrm{~nm}$ wavelength, and a laser focal position of -100 micron, i.e., with a focal position in front of the target surface, at 100 micron distance. This last requirement may induce self-focusing effect with spot decreasing on the target and consequent increase of the laser intensity. The target thickness is optimized to produce the maximum acceleration field, in fact decreasing or increasing the target thickness the acceleration effect is reduced, probably due to an electron density reduction. Lower thicknesses decrease the plasma electron density due to the lower number of involved nano-structures; higher thickness may increase the electron scattering decreasing the electron density along the normal direction, in agreement with previously measurements (Fucsh et al., 2006; Torrisi et al., 2013).

Thus, observing the literature data reporting the trend of the ion acceleration value as a function of the $I \lambda^{2}$ parameter, our measurements demonstrate that nonlinear effects induced by "advanced" targets can ensue the increasing of about two orders of magnitude the expected acceleration values. Figure 6 shows the law scale plot by Spencer et al. (2001) and, for comparison, our measurements, obtained by varying the laser pulse energy between 550 and $600 \mathrm{~J}$, the focal position between 0 and $-100 \mu \mathrm{m}$ and using "advanced targets" with thickness ranging between 10 and 15 micron. The comparison indicates that the use of special advanced high absorbent targets increases the ion acceleration of about one order of magnitude, i.e., to values normally expected only using fs lasers at intensity higher than $10^{18} \mathrm{~W} / \mathrm{cm}^{2}$. The maximum proton energy is reported as a function of the $I \lambda^{2}$ parameter; data from Tan et al. (1984) are denoted by circles, Beg et al. (1997) by triangles, and Clark and Al (2000) by diamonds.

\section{CONCLUSIONS}

The ion acceleration process in TNSA conditions depends not only on the laser intensity and wavelength, and not only on the irradiation conditions, such as the distance of laser focalization from the target surface, which is crucial, but also on the target composition and geometry. Advanced targets based on the surface treatment to decrease the reflection coefficient, on the use of embedded nano-structures enhancing the absorption coefficient and on the use of optimal thickness of the target, permit to increase the ion acceleration up to about one order of magnitude with respect to the planned values of literature data.

The obtainment of high energetic ions from lasergenerated plasmas represents only one step of the goal that would like to obtain about mono-energetic ions, high emission yields and selected ion specie emission.

Work is in progress to use polarized laser light at special incidence angles, in order to induce resonant absorption effects and increase the driving ion acceleration, and on the target geometry, using not flat surfaces, in order to increase the ion emission directivity decreasing the beam emittance of the accelerated ions. 


\section{ACKNOWLEDGEMENTS}

The author thank Dr. J. Ullschmied, Dr. E. Krousky, Dr. M. Pfeifer, Dr. J. Skala, and the staff of the PALS facilities in Prague for the use of the high intensity laser, plasma diagnostics, and high competent assistance, and the LaserLab-Europe to have accepted and financed the International proposal N. PALS-001823.

\section{REFERENCES}

Badziak, J., Glowacz, S., Jablonski, S., Parys, P., Wolowski, J. \& Hora, H. (2005). Laser-driven generation of high-current ion beams using skin-layer ponderomotive acceleration. Laser Part. Beams 23, 401-409.

Beg, F.N., Bell, A.R., Dangor, A.E., Danson, C.N., Fews, A.P., Glinsky, M.E., Hammel, B.A., Lee, P., Norreys, P.A. \& TATARAKIs, M. (1997). A study of picosecond laser-solid interactions up to $10^{19} \mathrm{~W} \mathrm{~cm}^{-2}$. Phys. Plasma 4, 447-457.

Clark, E.L., Krushelnick, K., Zepf, M., Beg, F.N., Tatarakis, M., MachaceK, A., Santala, M.I.K., Watts, I., Norreys, P.A. \& DANGor, A.E. (2000). Energetic heavy-ion and proton generation from ultraintense laser-plasma interactions with solids. Phys. Rev. Lett. 85, 1654-1657.

Cutroneo, M., Musumeci, P., Zimbone, M., Torrisi, L., La Via, F., Margarone, D., Velyhan, A., Ullschmied, U. \& Calcagno, L. (2012). High performance $\mathrm{SiC}$ detectors for $\mathrm{MeV}$ ion beams generated by intense pulsed laser plasmas. J. Mater. Res. 28, 87-93.

Dombi, P., Racz, P. \& Bodi, B. (2009). Surface plasmon enhanced electron acceleration with few-cycle laser pulses. Laser Part. Beams 27, 291-296.

Fucsh, J., Antici, P., D’Humieres, E., Lefebvre, E., Borghesi, M., Brambrink, E., Cecchetti, C.A., Kaluza, M., Malka, V., Manclossi, M., Meyroneinc, S., Mora, P., Schreiber, J., Toncian, T., Pepin, H. \& Audebert, P. (2006). Laser-driven proton scaling laws and new path towards energy increase, Nat. Phys. 2, 48-54.

Giulietti, D. \& Gizzi, L.A. (1998). X-ray emission from laser produced plasmas. La Rivista del Nuovo Cimento 21, 1-103.
Hegelich, B.M., Albright, B.J., Cobble, J., Flippo, K., Letzring, S., Paffett, M., Ruhl, H., Schreiber, J., Schulze, R.K. \& J.C. FERNANDEZ, J.C. (2006). Laser acceleration of quasimonoenergetic MeV ion beams. Nat. 439, 441-444.

Margarone, D., Klimo, O., Kim, I.J., Prokupek, J., Limpouch, J., Jeong, T.M., Mocek, T., Psikal, J., Kim, H.T., Proska, J., Nam, K.H., Stolcova, L., Chol, I.W., Lee, S.K., Sing, J.H., YU, T.J. \& KoRN, G. (2012). Laser-driven proton enhancement by nanostructured foils, Phys. Rev. Lett. 109, 234801/1-5.

McKenna, P., Lindau, F., Lundh, O., Neely, D., Persson, A. \& WAHLSTROM, C.G. (2006). High-intensity laser-driven proton acceleration: influence of pulse contrast, Phil. Trans. R. Soc. A 364, 711-723.

OPERA 3D TOSCA CODE. (2013). http://www.himarcsimulations.com/wp-content/Tosca_data_sheet.pdf.

Spencer, I., Ledingham, K.W.D., Singhal, R.P., McCanny, T., McKenna, P., Clark, E.L., Krushelnick, K., Zepf, M., Beg, F.N., Tatarakis, M., Dangor, A.E., Norreys, P.A., Clarke, R.J., Allott, R.M. \& Ross, I.N. (2001). Laser generation of proton beams for the production of sort-lived positron emitting radioisotopes. Nucl. Instr. Meth. B 183, 449-458.

Tan, T.H., McCall, G.H. \& Williams, A.H. (1984). Determination of laser intensity and hot-electron temperature from fastest ion velocity measurement on laser-produced plasma. Phys. Fluids 27, 296-301.

Torrisi, L., Cutroneo, M., Andò, L. \& Ullschmied, J. (2013). Thomson parabola spectrometry for gold laser-generated plasmas. Phys. Plasmas 20, 023106/1-7.

Torrisi, L., Margarone, D., Laska, L., Krasa, J., Velyhan, A., Pfeifer, M., Ullschmied, J. \& Ryc, L. (2008). Self-focusing effects in Au-target induced by high power pulsed laser at PALS. Laser Part. Beams 26, 379-387.

Zschornack, G., Musiol, G. \& Wagner, W. (1986). Dirac-FockSlater X-Ray Energy Shifts and Electron Binding Energy Changes for All Ion Groud States in Element up to Uranium. Akademy der Wissenschaften DER DDR, Zentralinstitut fur Kernforschung Rossendorf bei Dresden. 